\title{
THE ROLE OF ALLERGIC RHINITIS IN THE DEVELOPMENT OF OTITIS MEDIA WITH EFFUSION
}

Ranjan Kumar Guru

1. Senior Resident. Department of ENT, VSS Medical College, Burla, Odisha.

\section{CORRESPONDING AUTHOR:}

Ranjan Kumar Guru

Senior Resident

Department of Ent,

VSS Medical College, Burla,

Sambalpur, Odisha, PIN- 768017.

E-mail: dr.rkguru@gmail.com

ABSTRACT: The significant incidence of atopy associated with otitis media with effusion (OME) has suggested a role of allergy in the pathogenesis of OME. Past studies implicated allergy in the pathogenesis of otitis media [OM]. Otitis media is a common condition, especially in children. Most episodes of OM were associated with an upper respiratory viral infection and are shortlived and self-limiting with or without medical treatment. However, chronic OM with effusion (OME) has significant sequelae, is refractory to most medical treatments, and frequently requires surgical intervention. A large body of epidemiologic and mechanistic evidence supports a role for allergic rhinitis [AR] as a risk for OM. To establish the association of allergic rhinitis with otitis media with effusion 100 children of age group 5 to 14 were taken, 50 children were suffering from otitis media[test group] \& 50 were healthy[control group]. These children were evaluated clinically \& a battery of laboratory tests was performed in all children to establish the prevalence of allergic rhinitis in them. Out of 50 children with otitis media 12 were suffering from allergic rhinitis \& out of 50 healthy children 3 were suffering from allergic rhinitis. Chi-square test was performed and the 'P0.05' value was found to be 0.0127 [uncorrected] and was highly significant. These findings indicate that prevalence of allergic rhinitis is more in persons suffering from OME as compared to healthy individuals.

KEY WORDS: Allergic rhinitis, Otitis media, Eustachian tube dysfunction.

INTRODUCTION: Otitis media (OM) is a common and costly medical condition, especially in children. Otitis media (OM) is one of the most common chronic infectious diseases worldwide, affecting people in not only developing but also industrialized countries. It was defined as a chronic inflammation of the middle ear cleft. The significant incidence of atopy associated with otitis media with effusion (OME) has suggested a role of allergy in the pathogenesis of OME. Analysis of inflammatory mediators indicates that the mucosa of the middle ear can respond to antigen in the same way as does the mucosa of the lower respiratory tract. Recent characterization of the mucosa and effusion from atopic patients with OME reveals a Th2 cytokine, and cellular profiles consistent with an allergic response, supporting the role of allergy in OME [1]. The pathogenesis of otitis media with effusion (OME) was considered multifactorial, with viral upper respiratory tract infection and eustachian tube dysfunction. Allergy may be related to the pathogenesis of OME as an etiological factor [2]. Nonetheless, given the strong likelihood of allergy as a risk factor for OM, allergic rhinitis patients should be evaluated for OM and patients with OME should be considered for an allergy evaluation. If significant allergic 
rhinitis is diagnosed in a patient with OME, it should be treated aggressively (as in any case of moderate to severe allergic rhinitis) until further studies are conducted. No definitive conclusions about a role for food allergy in causing or treating OM can be made; hence more studies are needed to examine the relationship between these two important conditions [3]. The present study is an attempt to investigate the relationship between allergic rhinitis \& otitis media with effusion.

MATERIAL \& METHOD: The study was carried out from September 2008 to August 2010 in S.C.B. Medical College Cuttack, Odisha, among the children attending the Dept. of E.N.T. The total no. of children of age group 5 to 14 suffering from otitis media with effusion in this time period were 1040. Out of them 50 children were selected randomly after a thorough history taking \& clinical examination, these children constitute the test group. Another 50 healthy children of similar age group \& sex who were not suffering from otitis media were selected from nearby S.C.B. Public school, they constitute the control group.

A prospective case control cross-sectional study was carried out in these 100 children. The study protocol was fully explained to patients and controls, and written informed consent was obtained from their guardians.

For both test \& control groups, a detailed medical history was obtained, and physical examinations including anterior rhinoscopy, posterior rhinoscopy, otoscopy, tuning fork tests, impedance audiometry, pure tone audiometry, and speech audiometry, as indicated, were performed. Various blood tests were done to detect allergy.

Subjects with otitis media with effusion were diagnosed by presence of two or more of the following symptoms \& signs.

SYMPTOMS:- Blocking sensation of ear, conductive type deafness, wooly feeling in the ear, autophony, poor school performance.

SIGNS :-Dull or lusterless or congested tympanic membrane, air fluid level in middle ear, reduced mobility of tympanic membrane, tuning fork test - Rinne negative, Weber- lateralised to affected side, Absolute bone conduction test - normal.

Both groups were evaluated for the presence of allergic rhinitis. The criteria for diagnosis of allergic rhinitis were symptoms such as sneezing, watery rhinorrhoea, nasal obstruction, \& nasal or conjuctival itching (according to standard questionnaire) [4], and pale or watery nasal mucosa as detected by physical examination.

All the subjects were examined clinically at first, then they were subjected to the following tests.

1. Blood eosinophil count - Though eosinophil count was not always specific for allergic rhinitis subjects having eosinophil count more than 8 were considered to suffer from allergic diathesis.

2. Absolute eosinophil count[AEC] - persons having AEC more than 400 were considered to suffer from some allergic diathesis. 
3. Eosinophil count of nasal smear - normally no eosinophil should be present in nasal smear. Subjects having eosinophils in nasal smear were considered to be suffering from allergic rhinitis.

4. Total IgE - Venous blood was collected from cases and centrifuged at 2,500 rpm for $10 \mathrm{~min}$. Serum samples were stored at $-20^{\circ} \mathrm{C}$ until the analysis. Total IgE was determined in serum samples in duplicate with a commercially available enzyme linked immunoassay kit . Based on the kit's manual, all values higher than $100 \mathrm{IU} / \mathrm{ml}$ considered as high total IgE. Subjects having high IgE were considered to be suffering from allergic diathesis.

OBSERVATIONS: After comparing all clinical examination \& laboratory tests it was found that out of 50 children suffering from otitis media with effusion[test group], 12 children[24\% were suffering from allergic rhinitis \& out of 50 control subjects only 3 children[6\%] were suffering from allergic rhinitis.

The mean level of IgE in cases and controls were 105 and $82 \mathrm{IU} / \mathrm{ml}$, respectively. The mean level of IgE in the allergic rhinitis group compared to non allergic rhinitis was 138 and 70, respectively.

The children suffering/not suffering, from allergic rhinitis \&/or otitis media in our study is given in the underlying tables along with graphical illustrations.

TABLE: - 1

\begin{tabular}{|l|l|l|l|}
\hline OME & OME with AR & HEALTHY & HEALTHY with AR \\
\hline 50 & 12 & 50 & 3 \\
\hline
\end{tabular}

TABLE:-2

\begin{tabular}{|l|l|l|l|l|}
\hline \multirow{2}{*}{} & \multicolumn{4}{|l|}{ Otitis media with effusion } \\
\cline { 2 - 5 } & & Present & Absent Total \\
\hline \multirow{3}{*}{$\begin{array}{l}\text { Allergic } \\
\text { rhinitis }\end{array}$} & Present & 12 & 3 & 15 \\
\cline { 2 - 5 } & Absent & 38 & 47 & 85 \\
\cline { 2 - 5 } & Total & 50 & 50 & 100 \\
\hline
\end{tabular}

In our study $24 \%$ of cases were suffering from allergic rhinitis \& $6 \%$ controls were suffering from allergic rhinitis. Statistical analysis was done using Statcalc of Epi info version- 6. The Odd's ratio was 4.95 ( $\mathrm{CI}=1.17-23.97)$ and was statistically significant. Chi-square test was performed. Chi-square was found to be 6.35 which was significant, and the 'P0.05' value was found to be 0.0127 [uncorrected] and was highly significant.

DISCUSSION: Identifying the risk factors and pathogenesis of OME, it seems that the pathogenesis was multifactorial. Anatomical and functional characteristics of the eustachian tube were proposed to be the most important factors. Although the effect of allergic rhinitis on the eustachian tube function was well established, there was still a lack of evidence on the effect of the allergic rhinitis in development of the OME.

Numerous epidemiologic studies have identified allergy as a risk factor for otitis media with effusion [OME]. Alles et al. [5] reported an 89\% prevalence of allergic rhinitis among OME patients which was significantly higher than the reported prevalence of allergic rhinitis in the general population [6, 7]. In other study, otologic symptoms were reported in $32.8 \%$ of the 
children with allergic rhinitis. Comparing otologic and nasal symptoms showed, a significant association between allergic rhinitis and otitis media was found [8].

The allergic assessment tests such as serum total IgE concentration have some limitations; it was high in adults and children with allergic rhinitis and asthma, whereas many patients with atopy have serum IgE concentrations lower than normal individuals; therefore, it was not a useful parameter for screening atopic diseases [9]. In the current study, IgE was also used as a complementary test in cases who had a well known history of allergic rhinitis.

In a study by Caffarell et al[1998,Italy],the prevalence of allergic rhinitis was $16.3 \%$ in children with OME, while the prevalence of allergic rhinitis in healthy controls was 5.5\%[10].In a study by Kayhan et al,[2002,Turkey] the prevalence of allergic rhinitis in children suffering from OME was $23 \%$ \& the prevalence of allergic rhinitis in healthy controls was $4.8 \%$ [10].

In the present study, we used clear criteria for allergic rhinitis according to standard questionnaire and complementary tests and selected a matched-control group in children to enhance the validity of our study. There was a higher rate of allergic rhinitis among OME compared to controls, the difference was statistically significant. We know most of the OME initiate during childhood \{when the allergic rhinitis was more prevalent than adulthood [1113]\}, but it may present years later in the adulthood; therefore, in our survey there might be some recall bias during history taking for allergic rhinitis; however, there was such a bias for the controls as well.

In our study $24 \%$ of cases were suffering from allergic rhinitis \& $6 \%$ controls were suffering from allergic rhinitis. This values were comparable to the study done by Kayhan et al,[2002,Turkey] [10]. This difference between case \& control was statistically significant as the 'p0.05' value was 0.0127 using Epi info Version-6 Statcalc. The Odd's ratio was calculated to be 4.95 ( $\mathrm{CI}=1.17-23.97)$ and was found to be significant. Chi-square was found to be 6.35 which was significant. Thus it is presumed that children of the age group 5-14 years with history of allergic rhinitis are nearly 5 times more risk of contracting Otitis Media as compared to healthy children without such history.

Up to now, there was long way to explore more relevant factors which could finally prevent the morbidity and costs caused by OME, and more knowledge on its pathophysiology may provide new treatment avenues. However from above study it can be stated that 'allergic rhinitis has definitely some role in the development of otitis media with effusion'.

CONCLUSION: Otitis media was a condition with different possible etiologies. Applying strict diagnostic standard criteria for allergic rhinitis, we could show a significant difference ['p' value 0.0127] in the prevalence of allergic rhinitis in the affected children, as compared to the controls. These values were comparable to the previous study done by Kayhan et al. Hence we can conclude that allergic rhinitis has definitely some role in the development of Otitis media with effusion, or in other words patients suffering from Otitis media with effusion should be investigated to rule out allergic rhinitis. So patients with OME should also be prescribed treatment for allergic rhinitis.

\section{ABBREVIATIONS: \\ AR- Allergic rhinitis. \\ ET- Eustachian tube. \\ OM- Otitis media. \\ OME- Otitis media with effusion.}




\section{REFERENCES:}

1. Amber Luong PhD, MDa and Peter S. Roland MD $\underline{b}$ The Link Between Allergic Rhinitis and Chronic Otitis Media with Effusion in Atopic Patients Otolaryngologic clinics of north america, vol.41,issue2; April 2008, Pages 311-323.

2. Seung Geun A, Yeo. The role of allergic rhinitis in the development of otitis media with effusion: effect on eustachian tube function. Elsevier vol.28,issue-3,page 148-152; May2007.

3. Skoner, Amanda R, Kellen R, David P. Allergy \& Asthma Proceedings Ocean side publication,Inc. Vol.30, no:-5, Sept/Oct 2009, pp-470-481[12].

4. Bousquet. J, Reid J, Van Weel C et al[2008], Allergic rhinitis management pocket reference 2008. Allergy 63: 990-996.

5. Alles R, Parikh A, Hawk L et al (2001) The prevalence of atopic disorders in children with chronic otitis media with effusion.Pediatr Allergy Immunol 12(2):102-106.

6. Newacheck PW, Stoddard JJ (1994) Prevalence and impact of multiple childhood chronic illnesses. J Pediatr 124(1):40-48.

7. Sly RM (1999) Changing prevalence of allergic rhinitis and asthma. Ann Allergy Asthma Immunol 82(3):233-248.

8. Umapathy D, Alles R, Scadding GK (2007) A community based questionnaire study on the association between symptoms suggestive of otitis media with effusion, rhinitis and asthma in primary school children. Int J Pediatr Otorhinolaryngol 71(11):705-712.

9. Zeiss CR, Pruzansky JJ (1997) Immunology of IgE-mediated and other hypersensitivity states. In: Patterson R, Grammer LC,Greenberger PA (eds) Allergic disease: diagnosis and management 5th edn. Lippincott-Raven, Philadelphia, pp 27-40.

10. Medline study in Departments of Pediatrics, Catholic University of Rome and San Camillo De Lellis Hospital, Rome - Italy[2004].

11. Settipane RA (2003) Rhinitis: a dose of epidemiological reality. Allergy Asthma Proc 24(3):147-154

12. Druce H (1998) Allergic and nonallergic rhinitis. In: Middleton E, Reed CE, Ellis EF, Adkinson NF Jr, Yunginger JW, Busse WW (eds) Allergy principles and practice, 5th edn. Mosby-Year Book, St Louis, pp 1005-1016.

13. Smith JM (1971) A five-year prospective survey of rural children with asthma and hay fever. J Allergy 47:23-30. 


\section{ORIGINAL ARTICLE}

\section{GRAPH: - 1}

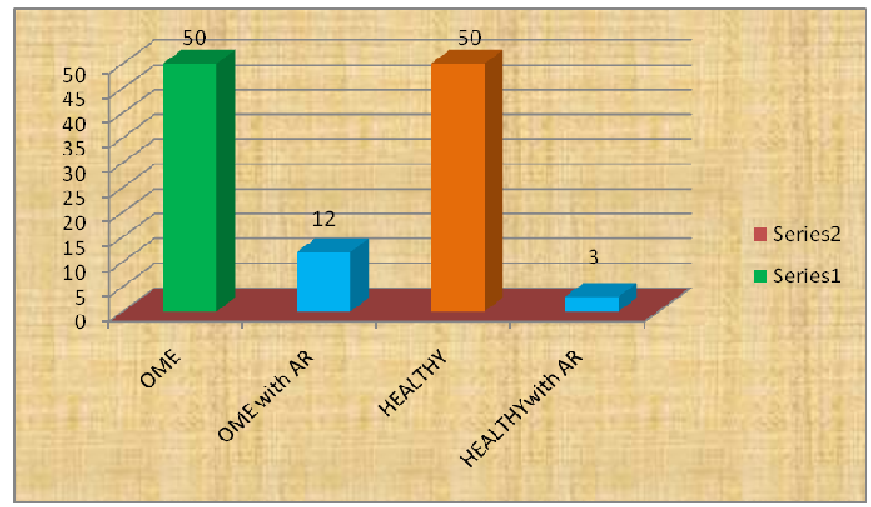

\section{GRAPH: - 2}

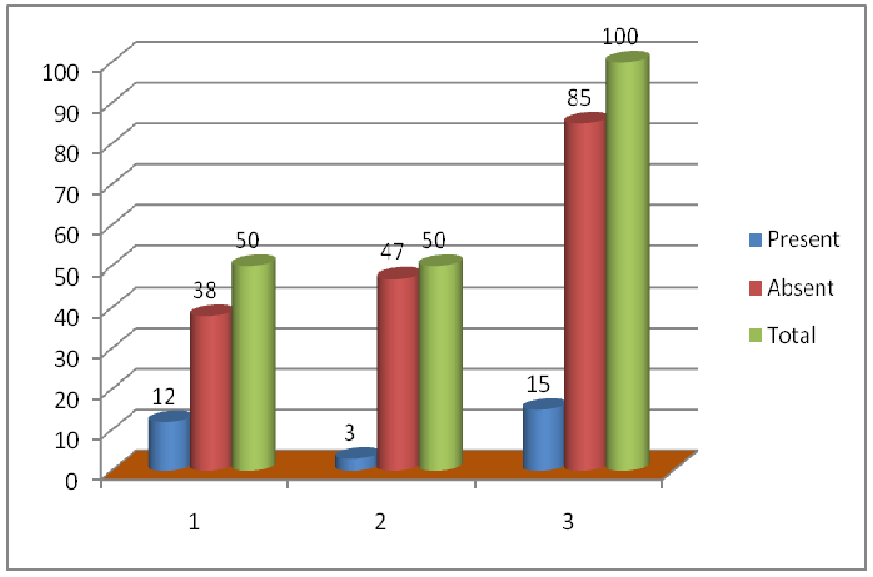

